\title{
Systemic design applied to water treatment for domestic purposes
}

\author{
D. Toso ${ }^{1} \& \mathrm{~T} . \mathrm{Re}^{2}$ \\ ${ }^{1}$ Politecnico di Torino (DAD), Italy \\ ${ }^{2}$ Università degli Studi di Genova, Italy
}

\begin{abstract}
The paper describes an innovative approach in researching water, developed within a PhD in "Production Systems and Industrial Design" at the Politecnico di Torino in collaboration with the UNESCO Chair in "Health Anthropology Biosphere and health therapies" at the Università degli Studi of Genova.

Specifically, the research is stretched according to the systemic design (Bistagnino 2011) methodology applied to water for domestic purposes by considering also the water benefits on human health according to health anthropology and ethnomedicine approaches. The research aims to design an alternative system of water treatment for domestic purposes able to enhance the qualities of water for specific uses by adopting alternative water treatment methodologies able to reduce the amount of water needed, the harmful byproducts production, and the re-use of wastewater. The starting point is the assessment of the complexity of water properties by considering it as an autopoietic system able to generate life and to sustain well being processes.

Recently, the understanding of the increasing complexity in the water structure, as a result of the discoveries which have occurred during the last century in physics and biology, made the reductionism view inadequate. The recent hypothesis about water structure according to quantum field theory (Montagnier, Del Giudice et al.) together with studies on its quality by scientists and naturalists (Schauberger, etc.) show us that water has essential properties for life which are not valued at present. Nature generates dynamic and autopoietic systems that improve themselves by maintaining their balance avoiding any kind of waste. According to these innovative theories, the Research Group aims to design an open water system for domestic purposes in which the quality of the
\end{abstract}


input and output water from each process will be enhanced and it will be used as an input for new activities.

Keywords: systemic design, alternative water treatment.

\section{Introduction}

Water is an essential resource for all life forms on the planet Earth. It is the most abundant molecular component of biological cells and, it may be argued, it's a pre-requisite for life is a likely requirement for pre-biotic evolution. Water covers the $71 \%$ of Earth's surface as well as the human body is made for the $65 \%$ of water (adult) and the $99 \%$ of our molecules are $\mathrm{H}_{2} \mathrm{O}$. Most of the water on our planet is represented by the oceans $(97.5 \%)$, while the glaciers and permanent snow store $1.72 \%$ of water resources [2].

However, the fresh water is not unlimited, like many of the natural resources that humanity is exploiting, such as oil and gas. The amount of fresh water generated from the water cycle is limited; although it can be reused, the water availability varies considerably in space and time and often it is not enough to satisfy human needs.

People use water mainly for drinking, cooking, cleaning, irrigate the soil, produce energy, as a fluid for productive activities, etc.

During the latest decades the global demand for drinkable water has been increased at a rate not sustainable due to the population growth rate and changes in the consumption model. At the same time, climate change, the overexploitation of the soil and deforestation directly influence the hydrological cycle and consequently the fresh water availability.

According to the Food and Agriculture Organization (FAO) [3] by 2050, 1.8 billion people will be living in countries or regions with absolute water scarcity, and two-thirds of the world population could be under stress.

Water scarcity and sustainable management of water resources therefore represent one of the largest commitments to the international community for the coming years. The global water availability is especially worrisome because it is subject to a trend that indicates a dramatic future if we are not able to change suddenly our approach on water management.

The world average of the water directly consumed for domestic purpose is about 137 liters per day per capita [4]. This quantity can be considerably greater (world average: 3,496 litres per capita per day) [5] if we consider the indirect water used for the production of products and food, the so called virtual water.

The virtual water needed for the growing of food, and for the production of products is often consumed outside the territory in which the product will be consumed. Therefore most of the water we consume is linked to the indirect footprint, which can affect the lives of people far away from the country in which the product is consumed. In Italy, more than $60 \%$ of the used virtual water comes from outside the territory [5].

Therefore the $\mathrm{PhD}$ research project is part of a wider scenario that involves the international scientific community in an effort to find immediate solutions to the global problem related to the scarcity of drinking water and its supply. 


\section{Methodology}

The methodological approach adopted by the $\mathrm{PhD}$ research is the systemic design, with the aim to look at the objective in its complexity by considering the water for its properties and qualities as an autopoietic system.

The systemic design approach (SD) aims to implement sustainable productive systems in which material and energy flows are designed so that waste from one productive process becomes input for other processes, avoiding being released into the environment. This model is inspired by the theoretical structure of generative science, according to which every modification in resources generates new products which represent added value.

Humans have always looked at nature as a model to answer their questions. systemic design approach aims to "learn from nature" to establish a change in the point of view in which we consider the environment. During the Industrial development the water supply chain has always been based on the intensive exploitation of natural resources (waters and chemicals) and consequently on the dangerous emissions to the environment. Rather the production activities should imitate the principles of the metabolic process of nature: living systems in constant change which jointly make progress, keeping their own typical relational structure.

In this way, systemic design plans open systems tending to zero emissions, in which the concept of waste does not apply anymore: any process output is fully employed as input in other processes. It is not an "end of pipe" approach oriented to limit the damage of a system no longer sustainable, but a radical change that releases opportunities and highlights perspectives, quite unthinkable only few decades ago [6]. The understanding of the rising complexity, resulting by the discoveries occurred during the last century in Physics and Biology, made the reductionist view inadequate: as a consequence the designer point of view moved from "the parts" to "all". The resulting design approach is then made up by the product design and the strategic design, and it is oriented to design and manage the entire product system taking into account the relationship with the environment and people (Tamborrini [7]).

The SD approach offers a design method that can be summed up by five basic principles:

- Output>input: the output (waste) of a system becomes the input (resource) for another one.

- Relationships: relationships generate the system because each one contributes to the system; they can be within the system or outside it.

- Auto-generation: self-producing systems sustain themselves by reproducing automatically, thus allow them to define their own paths of action and jointly co-evolve.

- Act locally: the local context is fundamental because it values local resources (humans, cultures and materials) and it helps to modify local problems in new opportunities.

- Man at the centre of the project: Man is connected to his own environmental, social, cultural and ethnic context. 
Thus SD can be profitably applied to the water treatment for its domestic purpose originating three main guidelines:

1. Output>input: the flows of output water (grey and black) can be collected and treated to become input, perhaps with different qualities, for the same house system, for other component of the "building system", or for different systems somehow related to them. The main goal is thus to re-use as much as possible its output as resources to reduce the income flows of drinkable water.

2. Relationship of the system: a house system acts as a living organism and is defined by relationships and flow exchanges of water (Montrucchio [8]). The water flow, within the house system, is constantly in relation with matter and substances and it is strictly related to the function has to solve.

3. Man at the centre of the project: the main scope of the drinkable water for its domestic purpose is to provide wellness to the human organism in a safe way. Therefore we need to deeper comprehend the water behaviour in the human body. This could represent the starting point to define the quality that drinkable water should have.

\section{Research}

Currently, the water for the domestic purpose is treated so as to be drinkable without particular distinction among its different functions (cooking, cleaning, toilet, etc.). Water is treated with the extensive use of chemicals during the disinfection and decontamination processes, it is carried through linear pipes and, at the end of the process; it is addressed to the sewerage system independently of its qualities.

The scientific community is focused to find more sustainable solutions in order to solve problems related to the water scarcity in a limited Planet where the population growth continues. The state of the art of the methods used to disinfect and decontaminate water and to reuse wastewater or to desalinate sea water, is working in concert to improve health, safeguard the environment, and reduce water scarcity. A revolution in water purification and treatment is now looking at both biological and physical solutions as for example RO membranes, membrane bioreactors (MBRs), photons and engineered nanostructures, etc. [9].

At the same time, there is a part of the Scientific Community involved in the better comprehension of what water really is. Ball, one of the premier science writers of our time, stated that no-one really understands water, and the stuff that covers two-thirds of our planet is still a mystery [10]. Therefore it could be crucial to acknowledge the variety of water properties and open the frontiers to a more holistic understanding of water. According to the SD approach the aim of the research is to assess the complexity of the qualities of water, by considering water as an autopoietic system able to generate life. Hence a deeper comprehension of water and its behaviour is needed to design innovative applications on the water treatment able to valorise the properties of this substance essential for life on planet Earth. 
Therefore, the crucial question on which this research is based concerns the true nature of water and its properties. Does the traditional way of water treatment able to preserve the whole qualities of water and its benefit for human health and the environment?

In order to optimize the use of water resources it is necessary to consider both the quantitative aspects (direct and indirect) and the qualitative characteristics of water we take from the environment, we use and we pour in nature again. It is commonly recognized that the quality of the water output is strictly connected to the quality of the water input and by the substances diluted into it during the various usage processes. This statement could sounds obvious, but still today the traditional approach on water management does not often take into consideration the whole properties of this incredible resource. Water functions as the medium for the most important biological processes, working as universal solvent for biomolecules, or as supplier of protons for charge transport [11].

Commonly, when it refers to the quality of water, the attention is focused on its chemical composition, its solvent abilities and the biological characteristics necessary to ensure life on the planet. However there is a less common recognized quality of water related to its energy content, that seems to have a great importance on the "water's social behaviour" and on its biological functions.

Starting from the XX century some research groups have dedicated their efforts in the investigation of the structure of water (Roentgen 1891; Bernal and Fowler 1933; Franck and Wen 1957; Nemethy and Sheraga 1962; Pople 1951; Mathouthi 1986; Mu Shik Jhon 2004; Chaplin 2000; Nilsson and Petterson 2004; Del Giudice and Preparata 1998; Stanley 2013 [12-18]). Some brilliant discoveries have resulted by showing a water interaction with electromagnetic fields. It has been discovered that the molecules of water, thanks to their bipolar character, are able to store the information through the creation of geometric structures and vibrating with specific frequencies.

The investigation of the structure of water has led to demonstrate that, even in the presence of liquid water can be found "crystals structure" as a result of ordered molecules.

$\mathrm{Mu}$ Shik Jhon [19] verified the existence of a specific structure of water, known as hexagonal water, which is found naturally in various parts of the world, particularly in some areas known for the longevity of the inhabitants. Jhon stated that the reinstatement of hexagonal water in our bodies, can increase vitality, slow aging and prevent disease.

Recently, Dr. Pollack, at the University of Washington, discovered the socalled Exclusion Zone in which the interfacial molecules of water assume a crystalline configuration very similar to ice structure. The interfacial water has been well known from many times, but Pollack observed a "solute free zone" extended about a tenth of a millimeter inward from the hydrophilic surface.

Recent studies are showing that water molecules have the ability to organize themselves into clusters or polymers based on electromagnetic fields which they are subjected to (Del Giudice et al. [19]). These stereo chemical configurations assume that the water mean that it becomes a carrier of information that 
influence the activity of the cells of living tissue. According to these theories, the Journal of Physics [20] published a notable research performed by two separate working groups, the first coordinated by Prof. Luc Montagnier, Nobel Prize for Medicine.

The research demonstrated how certain sequences of DNA can induce lowfrequency electromagnetic signals in highly diluted aqueous solutions, which then retain a "trace" of the characteristics of the DNA.

Similarly the experiments on activated water performed by Piccardi [21], led to recognize that water in temperatures where life is possible should be considered a quasi-crystal capable of assuming some specific conformations that could be "activated" by electromagnetic fields.

These incredible results could find a scientific explanation with the theories on quantum fields theory (QTF) elaborated by Del Giudice and Preparata [17] that led to the identification of biphasic structure in liquid water. The coherent phase described by Del Giudice could represent the crystalline structure of water identified by many research groups.

The coherent network of Coherence Domains make the water a candidate to be a dissipative system as described by Prigogine [23]. The existence of a dissipative structure consisting of a coherent set of Domains Consistency of water, whose consistency extended depends on the presence of guest non aqueous molecules in water, provides the ability of this liquid to communicate with the environment.

Liquid water has long been recognized as the matrix of many processes, including life and also the dynamics of the rocks. The interaction between biomolecules occurs differently from non-aqueous that are unable to produce life. This ability to make life processes implies a specific structure of liquid water (Del Giudice and Tedeschi [24]).

These innovative theories born in the medical and physical fields seem to confirm the intuitions advanced nearly a century ago by Schauberger and Schwenk about water behaviour.

Schauberger made an extraordinary contribution to knowledge of the natural world and particularly of the water. He intuited what modern discoveries of QFT recognise today as the quantum or subtle energy effects of water. His understanding was built up from experiential observation of nature in the untamed Alpine wilderness. His motto "Observe and Copy Nature" is according to the $\mathrm{SD}$ approach used in the $\mathrm{PhD}$ research. Viktor Schauberger's theories afford new insights into the naturally correct management of water by promoting its self-purification, the retention and enhancement of its natural energies and health.

The understanding of the raising complexity in water structure, resulting by the discoveries occurred during the last century in Physics and Biology, made the reductionist view inadequate. The recent hypothesis about the water structure according to quantum field theory, QFT (Montagnier et al. [20], Del Giudice et al. [19]) together with studies on its quality by scientists and naturalists (Schauberger [25], Schwenk, etc.) show us that water has properties essential for life which are not valued at present. 
Nature generates dynamic and autopoietic systems that improve themselves by maintaining their balance avoiding any kind of waste (Capra [26]). In nature, water flows from one system to another one regenerating continuously itself and transporting biological information necessary for life.

Regarding phenomena like the aforementioned interaction between water and electromagnetic fields and the essential function of the water in our cells we could actually be even more connected with this substance than we commonly think.

Therefore, the SD approach considers the water for its overall quality by considering water as "active" or "inactive" overcoming the traditional distinction between drinkable and not-drinkable water.

According to Schauberger, water is considered as a living organism. The vitality of water seems to be linked to its motion. Its ability to express natural flow patterns (like spiralling and meandering) determines whether water is dead or alive, healthy or dying. Vitality is quite probably related to the concept of energetic water quality (Alexandersson [27]).

As a consequence, the $\mathrm{PhD}$ research objective of optimizing the use of the water resource through the valorisation of its properties is aimed at investigating the whole qualities of water in order to assess a different use of it, in agreement with natural laws.

According to these innovative theories about the structure of water and its role in the biological processes the $\mathrm{PhD}$ research aims to design an open water system for domestic purpose in which the quality of the input and output water from each process will be enhanced and the water output will be used as an input for new activities in agreement with natural laws.

\section{Conclusion}

The first part of the research led to the definition of emergent properties of water seen as an open autopoietic system able to organize itself into domains of "super coherence".

Most of these theories, not all recognized by the international scientific community, now find direct application in the fields of medicine and homeopathy. The aim of this research is the combination of this knowledge to the context of water usage for domestic purposes.

In particular, the analysis phase highlights many points in common among the theories above mentioned that somehow validate them.

Specifically, it is possible to identify some general principles able to describe the properties of water that are useful to design a new water management flow for domestic purposes:

- Water has a two-phase structure consisting of a coherent state and an incoherent state.

- The temperature plays a key role in determining the quality of water. If the temperature is sufficiently low, there is order, but if it is high enough prevailing disorder. The maximum potential is obtained with water at $4^{\circ} \mathrm{C}$, in motion (centripetal) and placed in shadow. 
- The vortex is a key element in the regeneration and purification of water, but also to generate energy from movement and implosion. The water moves and it is regenerated through a curvilinear motion that is difficult to interface with sharp angles and straight pipes.

- The materials of pipelines that transport water can affect its quality, not only chemical quality, but also "structural" one.

- Water can be more or less "biologically active" according to the characteristics of its structure (clustered water).

- The water in our body has an organized structure. Scientific studies show that the structured water (clustered water) brings benefits to the body.

- Water responds to external electromagnetic influences, such as lowfrequency electromagnetic fields.

- Short ultraviolet radiation "turns on" the water. In the atmosphere this is masked by the ozone layer. Chlorophyll is able to transform long ultraviolet radiation into short ultraviolet.

- Water, especially in biological morphology, is a highly unstable system very sensitive to energy interactions.

- At the macro-level, water behaviour is related to biodiversity, the core of biological evolution.

The hypothesis advanced in this paper is based on the theory that water is an autopoietic system able to generate dissipative structures, in the form of supramolecular structures, structurally similar to a liquid crystal. Water is the most abundant substance on earth and has been very well studied with a number of model structures having been proposed and refined. Notwithstanding this, it remains an anomalous liquid where no single model is able to explain all of its properties.

\section{References}

[1] Bistagnino L., Systemic Design. Designing the productive and environmental sustainability. Slow Food ${ }^{\mathbb{B}}$ Editore srl, 2011

[2] UNESCO Water Portal, www.unesco.org/water

[3] FAO, Coping with water scarcity - Challenge of the twenty-first century, 2007

[4] Water Footprint Network, www.waterfootprint.org

[5] Mekonnen, M.M. and Hoekstra, A.Y., National water footprint accounts: the green, blue and grey water footprint of production and consumption, Value of Water Research Report Series No. 50, UNESCO-IHE, Delft, the Netherlands, 2011

[6] Toso D., Barbero S., Tamborrini P., Systemic Design: Beyond Ecodesign. In: 18th Annual International Sustainable Development Research Conference, University of Hull, 24-26 June 2012

[7] Tamborrini P.M., design sostenibile. oggetti, sistemi e comportamenti, Electa (ITA), pp. 1-224, Vol. 1, ISBN: 9788837055721, 2009 
[8] Montrucchio V., Systemic design approach applied to buildings. Analysis and comparison of international case studies. PhD thesis, 2013

[9] Mark A. Shannon, Paul W. Bohn, Menachem Elimelech, John G. Georgiadis, Benito J. Marinas \& Anne M. Mayes, Science and technology for water purification in the coming decades. Nature 452, pp. 301-310, 2008

[10] Ball, Philip, $\mathrm{H}_{2} \mathrm{O}$ : A Biography of Water. Weidenfeld \& Nicholson, 1999

[11] Voiekov V. L., Fundamental Role of Water in Bioenergetics, in Biophotonics and Coherent Systems in Biology, eds. L. V. Belussov, V. L. Voeikov and V. S. Martynyuk, Springer, New York, pp 89-104, 2007

[12] Rontgen, W.K. The structure of liquid water. Ann. Phys. 1892

[13] Silvestroni, Fondamenti di chimica, 10ª ed., CEA, 1996

[14] Pople, J. A. Molecular Association in Liquids: II. A Theory of the Structure of Water. Proceedings of the Royal Society A 205 (1081), 1951

[15] Chaplin, M. F., A proposal for the structuring of water. Biophys. Chemist., 83 (3), pp. 211-221, 2000

[16] Ph. Wernet, D. Nordlund, U. Bergmann, M. Cavalleri, M. Odelius, H. Ogasawara, L. Å. Näslund, T. K. Hirsch, L. Ojamäe, P. Glatzel, L. G. M. Pettersson and A. Nilsson, The structure of the first coordination shell in liquid water, Sciencexpress, 2004

[17] Del Giudice E., Preparata G. A new QED picture of water: understanding a few fascinating phenomena - in the volume Macroscopic Quantum Coherence, Singapore, 1998

[18] F. Mallamace, C. Corsaro, and H. E. Stanley, Possible Relation of Water Structural Relaxation to Water Anomalies, Proc. Natl. Acad. Sci. USA 110, pp. 4899-4904, 2013

[19] Del Giudice E., Stefanini P., Tedeschi A., Vitiello G., The interplay of biomolecules and water at the origin of the active behavior of living organisms. Journal of Physics: Conference Series 329, 012001, 2011

[20] Montagnier L., Aissa J., Del Giudice E., Lavallee C., Tedeschi A., and Vitiello G., DNA waves and water, J. Phys.: Conf. Ser. 306 012007, 2011

[21] Giorgio Piccardi, "The Chemical Basis of Medical Climatology", Springfield, Thomas, 1962

[22] Preparata G., QED Coherence In Matter, World Scientific, 1995

[23] Prigogine, I., The Behavior of Matter under Nonequilibrium Conditions: Fundamental Aspects and Applications in Energy-oriented Problems: Progress Report for Period September 1984-November 1987, Department of Physics at the University of Texas-Austin, United States, 1987

[24] Del Giudice E., Tedeschi A. - Water and the autocatalysis in living matter, Electromagnetic Biology and Medicine, 28, 46, 2009

[25] Coats C., The Schauberger Companion, ISBN 1-85860-011-1, 1994

[26] Capra F., The Web of Life: A New Synthesis of Mind and Matter Hammersmith, London: Harper Collins, 1996

[27] Alexandersson O., Living Water - Viktor Schauberger and the Secrets of Natural Energy, Gill and MacMillan, 2002 